\title{
QUALIDADE DE VIDA, SINTOMAS DEPRESSIVOS E DE ANSIEDADE NO INÍCIO DO TRATAMENTO QUIMIOTERÁPICO NO CÂNCER: DESAFIOS PARA O CUIDADO
}

Delma Aurélia da Silva Simão, Andreza Nayla de Assis Aguiar², Raissa Silva Souza, Karine Marley Captein², Bruna Figueiredo Manzo ${ }^{1}$, Antônio Lúcio Teixeira ${ }^{4}$

Objetivo: avaliar sintomas deansiedadee depressão e sua associação coma qualidade devida em pacientes antes da quimioterapia Métodos: estudo transversal, analítico, correlacional, com 55 pacientes. Utilizou-se questionário sociodemográfico e clínico, Escala Hospitalar de Ansiedade e Depressão (HADS) e escala de qualidade de vida Funcional Assessment of Cancer Therapy/ Gynaecologic Oncology Group - Neurotoxicity (FACT-GOG/Ntx.). Resultados: ansiedade 'provável' foi identificada em 2l,8\% da amostra, enquanto depressão 'provável' em 23,6\%. Houve correlação moderada e inversa para a maioria das subescalas e índices da FACT GOG/Ntx e os escores de ansiedade e depressão. Conclusão: observou-se relevante presença de sintomas de ansiedade e depressão antes da quimioterapia com impactos na sua qualidade de vida.

Descritores: Ansiedade, Depressão, Qualidade de Vida, Oncologia.

\section{QUALITY OF LIFE, SYMPTOMS OF DEPRESSIONAND ANXIETY ATTHE BEGINNING OF CHEMOTHERAPY CANCER TREATMENT: CHALLENGES TO CARE}

Objective: to assess symptoms of anxiety and depression and their association with quality of life in patients before chemotherapy. Methods: cross-sectional, analytical, correlational study with 55 patients. We used a sociodemographic and clinical questionnaire, Hospital Anxiety and Depression Scale (HADS) and to evaluate the quality of life on the Functional Assessment of Cancer Therapy / Gynecologic Oncology Group (FACT-GOG / Ntx.). Results: 'probable' anxiety was identified in $21.8 \%$ of the sample while 'probable' depression in $23.6 \%$. There was a moderate and inverse correlation for most FACT GOG / Ntx subscales and indexes and anxiety and depression scores. Conclusion: There was a significant presence of anxiety and depression symptoms before chemotherapy with impacts on their quality of life.

Descriptors: Anxiety, Depression, Quality of Life, Oncology.

\section{CALIDAD DE VIDA, LA ANSIEDAD Y LOS SÍNTOMAS DEPRESIVOS AL COMIENZO DE TRATAMIENTO QUIMIOTERÁPICO CONTRA EL CÁNCER: LOS RETOS DEL CUIDADO}

Objetivo: evaluar los síntomas deansiedad y depresióny su relación con la calidad devida de los pacientes antes de la quimioterapia. Métodos: Estudio transversal analítico, correlacional, con 55 pacientes. Se utilizó un cuestionario sociodemográfico y clínico, Hospital Anxiety and Depression Scale (HADS) y la escala de calidad de vida Evaluación Funcional de la Terapia del Cáncer / Grupo de Oncología Ginecológica - Neurotoxicidad (FACT-GOG/Ntx.). Resultados: la ansiedad de los "probables" fue identificado en el $21,8 \%$ de la muestra como la depresión "probable" en el 23,6\%. Hubo correlación moderada e inversa para la mayoría de las subescalas e índices FACT GOG / NTX y las puntuaciones de ansiedad y depresión. Conclusión: Hubo una presencia significativa de los sintomas de ansiedad y depresión antes de la quimioterapia con los impacto en su calidad de vida.

Descriptores: Ansiedad, Depresión, Calidad de Vida, Oncologia.

IEnfermeira. Doutora em Enfermagem. Professora da Universidade Federal de Minas Gerais - UFMG. ${ }^{2}$ Enfermeira. Mestranda - UFMG

${ }^{3}$ Enfermeira. Doutora em Enfermagem. Professora da Universidade Federal de São João Del Rei - UFSJ ${ }^{4}$ Médico. Doutor em Ciências. Membro do Laboratório Inter Disciplinar de Investigação. Medica da Faculdade de Medicina - UFMG 


\section{INTRODUÇÃO}

O câncer, em virtude de suas características etiopatogências e magnitude, tem se configurado como um importante problema de saúde pública na sociedade contemporânea. Parcela significativa de pessoas acometidas pelo câncer vivencia sintomas de ansiedade e depressão desde o diagnóstico da doença, os quais frequentemente persistem durante todo o tratamento, repercutindo na adesão ao tratamento, na qualidade de vida, no prognóstico e na sobrevida à doença ${ }^{(1-3)}$.

A depressão e a ansiedade são condições psiquiátricas manifestas por meio de sintomas psicológicos, comportamentais e físicos, decorrentes de uma combinação de múltiplos fatores. Tais condições podem se tornar crônicas ou recorrentes, acarretando prejuízos expressivos à capacidade da pessoa de se autocuidar e de realizar suas atividades diárias, impactando na sua qualidade de vida global ${ }^{(2,4)}$

Além disso, estudos evidenciam que a ansiedade e a depressão são mais frequentes entre pessoas com câncer do que entre aquelas com outras doenças crônicas, sendo que, quanto mais precocemente identificadas e abordadas, menor será o impacto na sua vida. Assim, a detecção e a abordagem multiprofissional dos sintomas de ansiedade e depressão são essenciais para a manutenção, restauração e/ou melhoria do bem estar das pessoas com câncer ${ }^{(2,5)}$.

Diante disso, este estudo tem por objetivo avaliar a presença de sintomas de ansiedade e depressão e sua associação com a qualidade de vida em pacientes com câncer antes de iniciar tratamento quimioterápico.

\section{METODOLOGIA}

Trata-se de estudo transversal, analítico e correlacional que incluiu pessoas com câncer e que iriam iniciar quimioterapia antineoplásica, atendidas em uma unidade funcional oncológica, ambulatorial, de um hospital universitário de Minas Gerais, entre fevereiro de 2013 e agosto de 2014

Aamostrafoi dimensionada deforma não probabilítistica, por conveniência, e foram incluídas todas as pessoas com câncer, com indicação de início da quimioterapia antineoplásica no período da coleta de dados e com idade superior a 18 anos.

Foram selecionadas 156 pessoas com indicação de quimioterapia no período estipulado, entretanto foram excluídos 101 indivíduos: com indicação de início imediato da quimioterapia $(n=10)$; com registros prévios de limitações cognitivas ( $n=9$ ); que não possuíam contato telefônico disponível ( $n=23)$; clinicamente debilitados e com prejuizo na capacidade funcional medida pela Escala de Performance da Eastern Cooperative Group - ECOG $(n=55)$; com metástases cerebrais e sequelas de acidente vascular encefálico ( $n=04 ; 1,5 \%)$.

Para a coleta dos dados, utilizou-se questionário de avaliação sociodemográfica e clínica; Escala Hospitalar de Ansiedade e Depressão (Hospital Anxiety and Depression Scale - HADS)(6) tendo em vista classificar os sintomas de ansiedade e depressão (improvável, provável ou possível); e o questionário de avaliação da qualidade de vida Funcional Assessment of Cancer Therapy/Gynaecologic Oncology Group - Neurotoxicity (FACT-GOG- Ntx) (7) na versão em português, no qual são avaliados 'bem estar físico', 'bem estar social/familiar', 'bem estar emocional', 'bem estar funcional' e sintomas de neurotoxicidade.

Os dados foram inseridos e analisados com o auxílio do software Statistical Package for the Social Sciences (SPSS 19.0), sendo utilizada estatística descritiva e analítica, considerando nível de significância de 5\% $(p<0,05)$. Para a análise descritiva das variáveis contínuas, foram realizados cálculos de suas medianas e intervalos interquartílicos (P25 e P75) e, para as variáveis categóricas, distribuições de frequências relativas e absolutas. Teste de Mann Whitney e análises de correlação de Spearman foram realizados para comparar os grupos com provável depressão e provável ansiedade aos escores das subescalas de qualidade de vida, assim como de seus índices. A interpretação da magnitude das correlações seguiu a classificação: coeficientes de correlação < 0,4 (correlação de fraca magnitude), > 0,4 a < 0,5 (de moderada magnitude) e > 0,5 (de forte magnitude).

A pesquisa foi analisada e aprovada pelo Comitê de Ética em Pesquisa com Seres Humanos (CAAE 08166912.8.0000.5149), sendo assegurados todos os direitos dos participantes.

\section{RESULTADOS}

Aidade dos participantes variou de 32 a 80 anos (mediana 54 anos), a maioria era do sexo feminino ( $n=46,83,6 \%)$, casada ( $n=25,45,5 \%)$ e com até 3 filhos ( $n=37,67,3 \%)$, com baixo nível de escolaridade ( $n=40,72,7 \%)$. Houve predomínio daqueles que se autodenominavam católicos ( $n=29,52,7 \%)$, que nunca fumaram ( $n=27,49,1 \%)$, que nunca beberam $(n=25,45,5 \%)$ e que se encontravam licenciados do trabalho $(n=26,47,3 \%)$. Além disso, a maioria dos participantes tinha prescrição de tratamento quimioterápico com esquemas terapêuticos envolvendo o paclitaxel ( $n=35,63,6 \%)$, em caráter adjuvante, sendo o câncer de mama predominante na amostra ( $n=32,60,4 \%)$.

Apesar dos escores da HADS apresentarem três índices ('improvável', 'provável' ou 'possível'), as pessoas que compuseram a amostra do presente estudo foram 
categorizadas em apenas dois desses escores - 'improvável' e 'provável'.

Em relação aos sintomas de ansiedade, 21,8\% dos participantes $(n=12)$ foram enquadrados no grupo de ansiedade 'provável'. De modo geral, os qualificadores com maior peso na caracterização da ansiedade pelos participantes foram "se sente tenso e contraído" $(n=34$, $61,8 \%)$, "fica com a cabeça cheia de preocupações" ( $n=49$, $89,1 \%$ ) e "se sente inquieta(o), como se não pudesse ficar parada (o) em nenhum lugar" ( $n=38,69,1 \%)$. Em relação aos sintomas depressivos, $23,6 \%(n=13)$ dos participantes foram enquadrados no grupo de depressão 'provável', sendo que os qualificadores mais indicados foram "não sentir gosto pelas mesmas coisas de antes" ( $n=28,51,1 \%)$, e "está lenta (o) para pensar e fazer as coisas" ( $n=31,56,4 \%$ ).

As comparações entre os grupos de pacientes com ansiedade 'improvável' $(n=43)$ e 'provável' $(n=12)$ e as subescalas e os índices de qualidade de vida total da
FACT GOG/Ntx evidenciaram a existência de diferença significativa entre os grupos em quase todos os quesitos. Assim, pode-se afirmar que pessoas com diferentes niveis de ansiedade têm impacto significativo no seu bem estar físico, funcional, emocional, social/familiar, bem como na sua qualidade de vida global.

Da mesma forma, as comparações entre os grupos com depressão 'improvável' (n=42) e 'provável' (n=13) e as subescalas de qualidade de vida da FACT GOG/Ntx demonstraram a existência de diferença estatisticamente significativa nas dimensões do bem estar físico, emocional, funcional, assim como na qualidade de vida global. Contudo, nesse caso, não houve associação entre os grupos e a subescala de bem estar social/familiar, o que pode indicar que a família e amigos oferecem suporte adequado nos momentos que antecedem o início da quimioterapia na amostra. Os dados referentes à comparação entre os grupos encontram-se apresentados na Tabela 1.

Tabela 1 - Ansiedade, depressão e comparação com a qualidade de vida em pacientes oncológicos antes da quimioterapia antineoplásica. Belo Horizonte,MG,Brasil, 2014(n=55).

\begin{tabular}{|c|c|c|c|c|c|c|}
\hline Parâmetros & $\begin{array}{l}\text { Ansiedade } \\
\text { provável ( } n=12) \\
\text { Mediana } \\
\text { (intervalo } \\
\text { interquartílico) }\end{array}$ & $\begin{array}{l}\text { Ansiedade } \\
\text { improvável } \\
(\mathrm{n}=43) \\
\text { Mediana } \\
\text { (intervalo } \\
\text { interquartílico) }\end{array}$ & Valor $\mathrm{p}^{*}$ & $\begin{array}{l}\text { Depressão } \\
\text { provável ( } n=13 \text { ) } \\
\text { Mediana } \\
\text { (intervalo } \\
\text { interquartilico) }\end{array}$ & $\begin{array}{l}\text { Depressão } \\
\text { improvável ( } n=42) \\
\text { Mediana } \\
\text { (intervalo } \\
\text { interquartílico) }\end{array}$ & Valor $p^{*}$ \\
\hline $\begin{array}{l}\text { Subescala de bem estar } \\
\text { físico }\end{array}$ & $21(14-22)$ & $26(22-28)$ & 0,001 & $22(11-24,5)$ & $25,5(21,8-28)$ & 0,013 \\
\hline $\begin{array}{l}\text { Subescala de bem estar } \\
\text { social/familiar }(0-28)\end{array}$ & $16(11,5-17,8)$ & $19(12-22)$ & 0,023 & $15(10,5-19,5)$ & $18(14,8-21)$ & 0,074 \\
\hline $\begin{array}{l}\text { Subescala de bem estar } \\
\text { mocional }(0-24)\end{array}$ & $14(9,3-17)$ & $20(18-22)$ & $<0,001$ & $15(11,5-18,5)$ & $20(17,8-22)$ & 0,008 \\
\hline $\begin{array}{l}\text { Subescala de bem estar } \\
\text { funcional }(0-28)\end{array}$ & $14(10-15,8)$ & $20(16-22)$ & $<0,001$ & $10(7,5-17,5)$ & $20(16-22)$ & 0,001 \\
\hline $\begin{array}{l}\text { Subescala de } \\
\text { neurotoxicidade }(0-44)\end{array}$ & $38(33,8-41)$ & $42(40-44)$ & 0,001 & $38(32-41)$ & $42,5(40,8-44)$ & $<0,001$ \\
\hline $\begin{array}{l}\text { Índice de } \\
\text { qualidade de vida (TOI) } \\
(0-100)\end{array}$ & $70,5(58-78,8)$ & $89(79-92)$ & $<0,001$ & $72(52-82,5)$ & $88,5(79-91,3)$ & $<0,001$ \\
\hline $\begin{array}{l}\text { Índice de } \\
\text { qualidade de vida total } \\
(0-108) \text { (FACT-G) }\end{array}$ & $61(45,8-70,8)$ & 81 (73-91) & $<0,001$ & $58(46,5-70,5)$ & $80(72,5-90,3)$ & $<0,001$ \\
\hline $\begin{array}{l}\text { Índice total de qualida- } \\
\text { de de vida (0-152) } \\
\text { (FACT/GOG-Ntx) }\end{array}$ & 99 (81,3-111) & 125(113-134) & $<0,001$ & $101(78,5-111)$ & $124(112,8-134)$ & $<0,001$ \\
\hline
\end{tabular}

Nota: *Teste de Mann Whitney, $\mathrm{p}<0,05$ 
Análises de correlação se mostraram de intensidade moderada e inversa para a maioria das subescalas e índices da FACT GOG/Ntx e os escores de ansiedade e depressão. Assim, à medida que aumentam os sintomas de ansiedade, pioram os níveis de qualidade de vida global, bem como o bem estar físico, funcional, emocional e social/familiar. Do mesmo modo, identificou-se que os sintomas de depressão pioram o bem estar físico, emocional, funcional e global dos pacientes. Os dados referentes à correlação entre os escores da Escala HAD e a Escala FACT GOG/Ntx encontram-se apresentados na Tabela 2 .

Tabela 2 - Correlação entre escores da FACT-GOG/Ntx e HAD em pacientes oncológicos anteriormente à quimioterapia. Belo Horizonte, MG,Brasil, 2014 ( $n=55)$.

\begin{tabular}{lll} 
Parâmetros & $\begin{array}{l}\text { Escore da } \\
\text { subescala de } \\
\text { ansiedade }\end{array}$ & $\begin{array}{l}\text { Escore da } \\
\text { subescala de } \\
\text { depressão }\end{array}$ \\
\hline $\begin{array}{l}\text { Subescala de bem } \\
\text { estarfísico }\end{array}$ & $\begin{array}{l}-0,464 \\
(<0,001)\end{array}$ & $-0,323(0,016)$ \\
\hline
\end{tabular}

$\begin{array}{lll}\begin{array}{l}\text { Subescala de bem } \\ \text { estar social/familiar }\end{array} & -0,232(0,088) & -0,402 \\ & & (0,002) \\ & & \\ \begin{array}{l}\text { Subescala de bem } \\ \text { estar emocional }\end{array} & -0,686 & -0,639 \\ & (<0,001) & (<0,001)\end{array}$

\begin{tabular}{|c|c|}
\hline $\begin{array}{l}\text { Subescala de bem } \\
\text { estar funcional }\end{array}$ & $-0,450(0,001)$ \\
\hline
\end{tabular}

$\begin{array}{lll}\text { Subescala de neuro- } & -0,424(0,001) & -0,405 \\ \text { toxicidade } & (0,002)\end{array}$

$\begin{array}{lll}\begin{array}{l}\text { Índice de qualidade } \\ \text { de vida (TOI) }\end{array} & \begin{array}{l}-0,495 \\ (<0,001)\end{array} & \begin{array}{l}-0,496 \\ (<0,001)\end{array} \\ & & \\ \begin{array}{l}\text { Índice de qualidade } \\ \text { de vida total }\end{array} & -0,573 & -0,631 \\ & (<0,001) & (<0,001)\end{array}$

$\begin{array}{lll}\text { Índice total de quali- } & -0,547 & -0,617 \\ \text { dade de vida } & (<0,001) & (<0,001)\end{array}$

Nota: Correlação de Spearman, $\mathrm{p}<0,05$
Podem-se observar correlações negativas, de moderada magnitude, especificamente entre o escore de ansiedade e as subescalas de bem-estar físico $(r=-0,464 ; p<0,001)$, bemestar funcional ( $r=-0,450 ; p=0,001)$. Quanto à relação entre a qualidade de vida e a depressão, tem-se correlação negativa de fraca magnitude apenas com a subescala de bem-estar físico $(r=-0,323 ; p=0,016)$.

\section{DISCUSSÃO}

Aanálise dos dados evidenciou que pouco mais de $20 \%$ dos participantes deste estudo apresentava 'provável' ansiedade e depressão antes do início do tratamento quimioterápico, corroborando com dados da literatura nos quais índices de ansiedade e de depressão em pessoas com câncer são de 20 a $48 \%{ }^{(5)}$. A existência dessas condições nessa população tem relação com seus temores em relação ao câncer, ao início do tratamento quimioterápico (com seus possiveis efeitos colaterais e alterações corporais) e ao seu impacto em sua qualidade de $v i^{(8)}{ }^{(8)}$. Além disso, salienta-se que as pessoas que recebem o diagnóstico de câncer e tomam ciência dos recursos disponiveis para seu tratamento, de maneira geral, passam por uma fase de ajustamento, quando os sintomas de ansiedade e depressão são frequentes, com tendência ao declínio quando conseguem integrar as novas informações à sua realidade, encontrando com isso, razões novas para prosseguir com sua vida(9)

Sugere-se que o reduzido percentual de participantes com 'provável' ansiedade e depressão, encontrado no presente estudo, possa ter relação com questões subjetivas como a religiosidade, que, de modo geral, encontra-se potencializada na vida de pessoas com câncer, e a resiliência que também pode estar presente no momento do diagnóstico do câncer e do início do tratamento ${ }^{(5,10)}$

Além disso, há indícios da participação do suporte social provido pela família, comunidade e equipe de saúde na redução dos níveis de ansiedade e depressão, sendo que esse suporte influencia positivamente na adesão ao tratamento, na adaptação psicossocial e nos mecanismos de enfrentamento da pessoa com câncer. Nesse sentido, sugere-se que o enfermeiro oncologista investigue, em sua prática cotidiana, a existência e a qualidade da rede de apoio social da pessoa com câncer ${ }^{(11,12)}$

No presente estudo identificou-se piora significativa na percepção da qualidade de vida dos pacientes com depressão e/ou ansiedade, quando comparados com pacientes sem depressão e/ou ansiedade. As correlações negativas entre os escores de ansiedade e os escores de 'bem-estar físico', 'bem-estar funcional' e 'bem-estar social/familiar' sugerem que a presença de sintomas ansiosos pode influenciar negativamente a percepção de sintomas físicos, da 
funcionalidade e do apoio social e/ou familiar recebido pelos participantes deste estudo. Corroboram com esse achado dados encontrados em outro estudo que demonstraram associação entre a ansiedade e os sintomas físicos, bem como piora na capacidade funcional da pessoa com câncer ${ }^{(5)}$.

Da mesma forma, as correlações negativas entre os escores de depressão e o escore de 'bem-estar físico' sugerem que a presença de sintomas depressivos pode influenciar negativamente a percepção de sintomas físicos das pessoas que compuseram a amostra. Estudos apontam que a depressão no paciente com câncer acarreta intensificação dos sintomas físicos e exacerbação das preocupações em relação à doença, às relações sociais e familiares e à situação financeira, piorando o enfrentamento adaptativo dessas pessoas $^{(5,9)}$

Tendo em vista esses resultados, destaca-se a importância da identificação e da abordagem multiprofissional da ansiedade e da depressão nos serviços de oncologia, enfocando não apenas os sofrimentos ocasionados por essas condições psiquiátricas, mas também sobre os seus efeitos sobre os diferentes domínios da qualidade de vida da pessoa com câncer. A detecção precoce e a intervenção eficaz junto às pessoas com sintomas de ansiedade e depressão podem reduzir o custo humano do câncer, não só para os pacientes, mas também para os cuidadores e/ou familiares ${ }^{(5)}$.

Acredita-se que a pesquisa ora apresentada comporte limites, destacando-se a impossibilidade de inferir direcionalidade ou causalidade a partir dos resultados obtidos, restrição própria de estudos com desenho transversal.

\section{CONCLUSÃO}

Observou-se a presença de sintomas de ansiedade e depressão na população oncológica antes da quimioterapia, com impactos na sua qualidade de vida. Dessa forma, sugere-se que a equipe de saúde, em especial o enfermeiro, atue ativamente na identificação precoce de tais sintomas, possibilitando, dessa forma, o estabelecimento de planos de cuidados individualizados com vistas à melhora da qualidade de vida das pessoas com câncer

\section{REFERÊNCIAS}

1. Bergerot CD, Laros JA, Araujo TCCF. Avaliação de ansiedade e depressão em pacientes oncológicos: comparação psicométrica. Psico-USF [Internet]. 2014 Maio/Ago; 19(2):187-197.

2. Souza BF, Pires FH, Dewulf NLS, Inocenti A, Silva AEBC, Miasso AI. Pacientes em uso de quimioterápicos: depressão e adesão ao tratamento. Rev. esc. enferm. USP [Internet]. 2013;47(1).

3. Paula JM, Sawada NO. Qualidade de vida relacionada à saúde de pacientes com câncer em tratamento radioterápico. Rev Rene [ Internet]. 2015 Jan/Fev; 16(1):106-13.

4. Santos CA, Ribeiro AQ, Rosa COB, Ribeiro RCL. Depressão, déficit cognitivo e fatores associados à desnutrição em idosos com câncer. Ciência \& Saúde Coletiva [Internet]. 2015; 20(3):751760.

5. Barbara LA, Robert JR, Barry SB, Jessie G, Victoria LC, Mary J M et al. Screening, Assessment, and Care of Anxiety and Depressive Symptoms in Adults With Cancer: An American Society of Clinical Oncology Guideline Adaptation. J Clin Oncol [Internet]. 2014 May; 32(15): 1605-1619.

6. Botega NJ, Márcia RB, Maria AZ, Celso GJ, Walter ABP. Transtornos do humor em enfermaria de clínica médica e validação de escala de medida (HAD) de ansiedade e depressão. Revista de Saúde Pública [Internet]. 1995 Out; 29(5): 355-363.
7. Cella D. Functional assessment of chronic illness therapy. Elmhurst: Facit [Internet]. 2010. Available from: http://www.facit. org/FACITOrg/Questionnaires

8. Organização Mundial de Saúde. Relatório sobre depressão. [Ministério da Saúde] [Internet]. 2014 [cited 2016 Ago 06]. Available from: http://abp.org.br/portal/clippingsis/ exibClipping/?clipping=18806

9. Terra FS, et al. Avaliação da qualidade de vida de pacientes oncológicos submetidos à quimioterapia. Rev Bras Clin Med. [Internet]. 2013; 11(2):112-7.

10. Albuquerque KA, Pimenta CAM. Distress do paciente oncológico: prevalência e fatores associados na opinião de familiares. Rev Bras Enferm [Internet]. 2014 Set/Out; 67(5):744-51.

11. Santos JFS, Lima ACR, Mota CMD, Gois CFL, Brito GMG Barreto ÍDC. Qualidade de vida, sintomas depressivos e adesão ao tratamento de pessoas com hipertensão arterial. Rev. Enferm. Foco [Internet]. 2016; 7(2):17-21.

12. Souza RS, SIMÃO DAS, LIMA EDRP. Perfil sociodemográfico e clinico de pacientes atendidos em um serviço ambulatorial de quimioterapia paliativa em Belo Horizonte. REME. 2012; 12(1):38-47. 